\title{
Partial Fraction Evaluation by an Escalation Technique
}

\author{
By J. F. Mahoney
}

\begin{abstract}
An escalation method for performing partial fraction expansions is presented for the case that the complete list of zeros of the denominator of the proper rational function is known. Expressions for the number of divisions and multiplications required are developed. The new method requires fewer such arithmetic operations than do other known methods. A numerical example is provided.
\end{abstract}

Introduction. At times it is desired to express a rational function in terms of partial fractions. After completely factoring the denominator polynomial into linear factors it is conceptually easy to perform the expansion, but more efficient methods of carrying out the calculation are always welcome. Particular attention is directed to the situation where the linear factors of the denominator occur repeated. The partial fraction expansion technique that is presented here is efficient relative to the number of arithmetic operations required. It compares favorably in this regard with method B of Henrici [2, p. 555].

The Escalation Process. Consider the proper rational function

$$
\phi(x)=\frac{P(x)}{Q(x)\left(x-\xi_{a}\right)^{A-1}\left(x-\xi_{b}\right)^{B}},
$$

where $\xi_{a} \neq \xi_{b}, A$ and $B$ are integers greater than zero, and $P(x)$ and $Q(x)$ are polynomials for which neither $\xi_{a}$ nor $\xi_{b}$ are zeros. In terms of partial fractions,

$$
\phi(x)=\sum_{i=1}^{A-1} \frac{C_{a i}}{\left(x-\xi_{a}\right)^{i}}+\sum_{i=1}^{B} \frac{C_{b i}}{\left(x-\xi_{b}\right)^{i}}+\chi(x) .
$$

Here and in other parts of this paper a summation is taken to vanish if its upper limit is less than its lower limit. A related function $\hat{\phi}(x)$ has the definition and partial fraction expansion given by

$$
\hat{\phi}(x)=\frac{\phi(x)}{\left(x-\xi_{a}\right)}=\sum_{i=1}^{A} \frac{\hat{C}_{a i}}{\left(x-\xi_{a}\right)^{i}}+\sum_{i=1}^{B} \frac{\hat{C}_{b i}}{\left(x-\xi_{b}\right)^{i}}+\hat{\chi}(x) .
$$

In going from $\phi(x)$ to $\hat{\phi}(x)$ the power of $\left(x-\xi_{a}\right)$ in the denominator was increased by unity. For this reason those $A$ partial fraction coefficients represented by $\hat{C}_{a i}$ are called the native coefficients of (3). All other partial fraction coefficients of (3) (the $\hat{C}_{b i}$ and those contained in $\hat{\chi}(x)$ ) are called alien coefficients. What follows will

Received February 12, 1980; revised May 27, 1980.

1980 Mathematics Subject Classification. Primary 65F99. 
explore and exploit the conjecture that the partial fraction coefficients (both native and alien) of (3) may be computed from their counterparts in (2).

First consider the equation

$$
\left(x-\xi_{a}\right)^{A-1} \phi(x)=\left(x-\xi_{a}\right)^{A} \hat{\phi}(x)
$$

and the $(A-2)$ equations obtained through repeated differentiation with respect to $x$ (if $A=1$, or 2 , no differentiation is indicated). When $x$ is set equal to $\xi_{a}$ one finds that

$$
\hat{C}_{a i}=C_{a, i-1}, \quad i=2,3, \ldots, A .
$$

No information is gained concerning $\hat{C}_{a 1}$.

Next consider

$$
\left(x-\xi_{b}\right)^{B} \phi(x)=\left(x-\xi_{b}\right)^{B}\left(x-\xi_{a}\right) \hat{\phi}(x)
$$

and the $(B-1)$ equations obtained through successive differentiation. Upon setting $x=\xi_{b}$ one finds that

$$
\hat{C}_{b B}=\frac{C_{b B}}{\xi_{b}-\xi_{a}}
$$

and

$$
\hat{C}_{b i}=\frac{C_{b i}-\hat{C}_{b, i+1}}{\xi_{b}-\xi_{a}}, \quad i=B-1, B-2, \ldots, 2,1 .
$$

Alternatively, (5) may be derived by expressing $\phi(x)$ and $\hat{\phi}(x)$ in Laurent series in the neighborhood of $\xi_{a}$ and then equating the coefficients of the series for $\phi(x)$ and $\left(x-\xi_{a}\right) \hat{\phi}(x)$. Similarly, (7) and (8) may be gained by forming the Laurent series for $\phi(x)$ and $\hat{\phi}(x)$ in the neighborhood of $\xi_{b}$ and then comparing the coefficients for the series $\phi(x)$ and $\left[\left(x-\xi_{b}\right)-\left(\xi_{a}-\xi_{b}\right)\right] \hat{\phi}(x)$.

Application of Escalation. Consider the proper rational function given by

$$
F_{n}(s)=\frac{N_{n}(s)}{D_{n}(s)}
$$

where

$$
N_{n}(s)=\sum_{i=0}^{n-1} b_{i} s^{i}
$$

and

$$
D_{n}(s)=\prod_{j=1}^{n}\left(s-s_{j}\right)=\prod_{j=1}^{q}\left(s-\sigma_{j}\right)^{M_{j}}
$$

The numerator $N_{n}(s)$ is a polynomial whose degree is no greater than $(n-1)$. The denominator $D_{n}(s)$ is a polynomial of degree $n$ and has been assumed to be monic (that is, the coefficient of $s^{n}$ has been taken to be unity). The distinct zeros of $D_{n}$ are $\sigma_{1}, \sigma_{2}, \ldots, \sigma_{q}$ which occur with multiplicities $M_{1}, M_{2}, \ldots, M_{q}$, respectively. The complete list of the zeros of $D_{n}(s)$ is $s_{1}, s_{2}, \ldots, s_{n}$, where it is convenient, but not necessary, to arrange the list so that the first $M_{1}$ items all equal $\sigma_{1}$, the next $M_{2}$ items all equal $\sigma_{2}$, and so forth until the final $M_{q}$ items all equal $\sigma_{q}$. In the event that the given denominator polynomial is not monic, one may first find the partial 
fraction coefficients for the monic case and then divide all of these coefficients by the actual coefficient of $s^{n}$ in order to get the desired partial fraction coefficients for the nonmonic case.

The numerator may be written using Newton's interpolation formula as

$$
N_{n}(s)=\beta_{0}+\sum_{i=1}^{n-1} \beta_{i} \prod_{j=1}^{i}\left(s-s_{j}\right)
$$

where the $\beta$ coefficients may be found using Horner's scheme. The details of this calculation will be illustrated later. Upon defining $N_{0}(s)=0, D_{0}(s)=1$, and

one obtains

$$
F_{r}(s)=\frac{\beta_{0}+\sum_{i=1}^{r-1} \beta_{i} \prod_{j=1}^{i}\left(s-s_{j}\right)}{\prod_{j=1}^{r}\left(s-s_{j}\right)}, \quad r=1,2, \ldots, n,
$$

$$
F_{r}(s)=\frac{F_{r-1}(s)}{\left(s-s_{r}\right)}+\frac{\beta_{r-1}}{\left(s-s_{r}\right)}, \quad r=1,2, \ldots, n .
$$

All of the $F_{r}(s)$ thus defined are proper rational functions and hence have partial fraction expansions.

The immediate aim is to find the partial fraction expansion of $F_{r}(s)$ from the corresponding expansion of $F_{r-1}(s)$. If this can be accomplished, it can be repeated $n$ times for $r=1,2, \ldots, n$, ultimately yielding the expansion for $F_{n}(s)$.

The process of going from the partial fraction expansion of $F_{r-1}(s)$ to that of $F_{r}(s)$ may be broken into steps. First define

$$
\hat{F}_{r}(s)=\frac{F_{r-1}(s)}{\left(s-s_{r}\right)}, \quad r=1,2, \ldots, n,
$$

and note that, if the expansion of $F_{r-1}(s)$ is known, with the exception of the first native coefficient, all of the partial fraction cofficients of $\hat{F}_{r}(s)$ may be found by properly invoking the notions embodied in (5), (7), and (8). Then, according to (14), $\beta_{r-1} /\left(s-s_{r}\right)$ is added to the foregoing result. This addition causes the alteration of only one coefficient in $F_{r}(s)$ as compared to $\hat{F}_{r}(s)$. That altered coefficient is the same initial native coefficient for which the method of escalation sheds no light. Thus, the escalation policy as given by (5), (7), and (8) is sufficient to transform the coefficients of $F_{r-1}(s)$ into all but one of the coefficients of $F_{r}(s)$. The remaining initial native coefficient of $F_{r}(s)$ may be found using the fact that the sum of the residues of any rational function, whose denominator degree exceeds its numerator degree by two or more, is zero [1]. Since $F_{r-1}$ is a proper rational function, this applies to $\hat{F}_{r}(s)$ and by (14) it is concluded that the residue sum of $F_{r}(s)$ is $\beta_{r-1}$.

Inspection of (13) reveals that the denominator of $F_{r-1}(s)$ must be augmented by the factor $\left(s-s_{r}\right)$ in order to arrive at the denominator of $F_{r}(s)$. Upon identifying $\alpha, 1 \leqslant \alpha \leqslant q$, such that $s_{r}=\sigma_{\alpha}$, one may write the partial fraction expansion of $F_{r}(s)$ as

$$
F_{r}(s)=\sum_{j=1}^{L(r)} \frac{B_{\alpha j}^{(r)}}{\left(s-\sigma_{\alpha}\right)^{j}}+\sum_{\substack{i=1 \\ i \neq \alpha}}^{q} \sum_{j=1}^{L \xi^{(r)}} \frac{B_{i j}^{(r)}}{\left(s-\sigma_{i}\right)^{j}}, \quad 1 \leqslant r \leqslant n .
$$


The $L_{k}^{(r)}, k=1,2, \ldots, q$, are integers which represent the multiplicities of the factors $\left(s-\sigma_{k}\right)$ in the denominator of $F_{r}(s)$; they sum to $r$ and are bounded in accordance with, $0 \leqslant L_{k}^{(r)} \leqslant M_{k}$. During the escalation process the multiplicities change one at a time so that

$$
L_{\alpha}^{(r-1)}+1=L_{\alpha}^{(r)}, \quad s_{r}=\sigma_{\alpha}, \quad 1 \leqslant \alpha \leqslant q,
$$

and

$$
L_{i}^{(r-1)}=L_{i}^{(r)}, \quad i=1,2, \ldots, q, i \neq \alpha .
$$

The process comes to completion when $r=n$ and $L_{k}^{(n)}=M_{k}, k=1,2, \ldots, q$.

In (16) one may identify those coefficients represented by $B_{i j}^{(r)}$ as being the alien coefficients, and those written as $B_{\alpha j}^{(r)}$ as the native coefficients. The alien coefficients of $F_{r}(s)$ may be formed from their counterparts in $F_{r-1}(s)$ by applying (7) and (8) which are now recast as

$$
B_{i j}^{(r)}=\frac{B_{i j}^{(r-1)}}{\sigma_{i}-\sigma_{\alpha}}, \quad j=L_{i}^{(r)} \geqslant 1,
$$

and

$$
B_{i j}^{(r)}=\frac{B_{i j}^{(r-1)}-B_{i, j+1}^{(r)}}{\sigma_{i}-\sigma_{\alpha}}, \quad j=L_{i}^{(r)}-1, \ldots, 2,1 .
$$

In (19) and (20), $i=1,2, \ldots, q, i \neq \alpha$, where $\alpha$ is found from $s_{r}=\sigma_{\alpha}$. The native coefficients of (16) may be calculated from (5) and from the notion that the residue sum is $\beta_{r-1}$. The resultant equations are

$$
B_{\alpha 1}^{(r)}=\beta_{r-1}-\sum_{\substack{i=1 \\ i \neq \alpha}}^{q} B_{i 1}^{(r)}
$$

and

$$
B_{\alpha j}^{(r)}=B_{\alpha, j-1}^{(r-1)}, \quad j=2,3, \ldots, L_{\alpha}^{(r)}
$$

For each value of $r$, starting with $r=1,(19),(20),(21)$, and (22) are used in that order. Coefficients not previously found are taken to be zero.

Illustration of the Method. For a more concrete presentation, attention is directed to the finding of the partial fraction expansion of

$$
F(s)=\frac{1+2 s+3 s^{2}+4 s^{3}+5 s^{4}+6 s^{5}}{24-104 s+182 s^{2}-164 s^{3}+80 s^{4}-20 s^{5}+2 s^{6}}=\frac{N_{6}(s)}{2 D_{6}(s)} .
$$

In a separate (and by no means trivial) calculation it may be found that: $\sigma_{1}=1$, $M_{1}=3 ; \sigma_{2}=2, M_{2}=2 ; \sigma_{3}=3, M_{3}=1$. This means that the complete list of zeros of $D_{6}(s)$ is: $1,1,1,2,2,3$. The $\beta$ values may be found by Horner's scheme, which involves the repeated use of synthetic division using the complete list of zeros of $D_{6}(s)$ as divisors. The initial dividend is the coefficients of $N_{6}(s)$ and the remainders are the $\beta$ coefficients The process in abbreviated form is now shown. 


$$
\begin{array}{rlllllllllr}
\underline{1} \mid & {[6} & , & 5 & , & 4 & , & 3 & , & 2 & 1 \\
\underline{1} \mid & {[6} & , & 11 & , & 15 & , & 18 & , & 20] & 21 \\
\underline{1} \mid & {[6} & , & 17 & , & 32 & , & 50] & & 70 & \\
\underline{2} \mid & {[6} & , & 23 & , & 55] & & 105 & & & \\
\underline{2} \mid & {[6} & , & 35] & & 125 & & & & & \\
\underline{3} \mid & {[6]} & & 47 & & & & & & &
\end{array}
$$

It may be verified that

$$
\begin{aligned}
N_{6}(s)= & 21+70(s-1)+105(s-1)^{2}+125(s-1)^{3} \\
& +47(s-1)^{3}(s-2)+6(s-1)^{3}(s-2)^{2}
\end{aligned}
$$

Next, use is made of the tableau given in Figure 1.

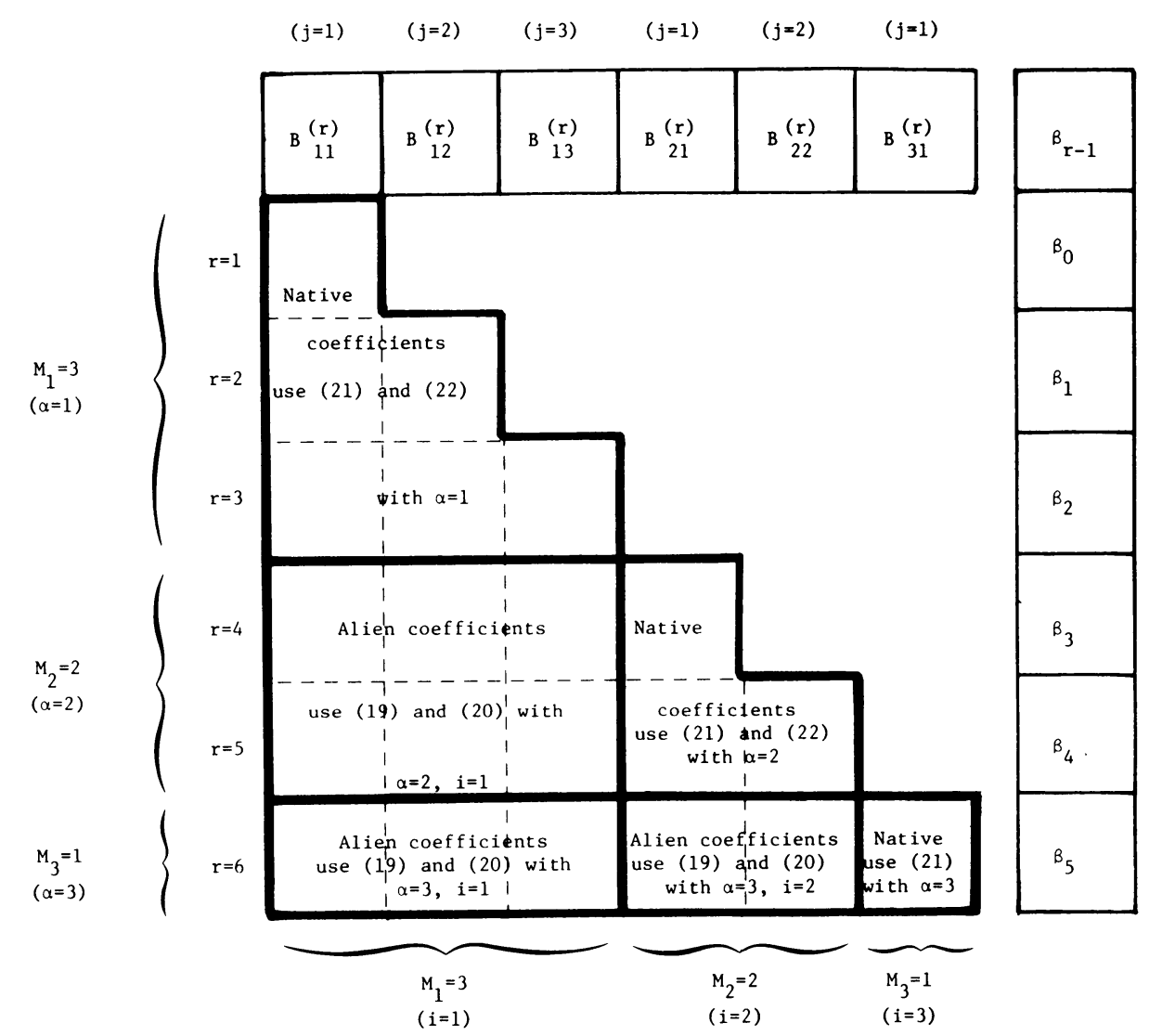

Figure 1

Tableau used in computing the $B_{i j}^{(r)}$ coefficients

The blank spaces in each row of Figure 1 represent locations where the partial fraction coefficients of $F_{r}(s), r=1,2, \ldots, 6$, are to be placed. The tableau is to be filled in from the top to the bottom, with each successive row being computed from the row above and from the $\beta$ coefficient to the right of the row in question. In particular, those regions labeled alien coefficients are to be filled in using (19) and (20), while the native coefficients are formed using (21) and (22). 
Omitting the details of the calculation, the entries in the tableau are:

$\begin{array}{rrrrrrr}21 & & & & & & 21 \\ 70 & 21 & & & & 70 \\ 105 & 70 & 21 & & & \\ -196 & -91 & -21 & 321 & & 105 \\ 308 & 112 & 21 & -261 & 321 & 125 \\ -\frac{1477}{8} & -\frac{245}{4} & -\frac{21}{2} & -60 & -321 & \frac{2005}{8} & 47\end{array}$

Since the denominator of $F(s)$ is not monic (the coefficient of $s^{6}$ is 2 ), all of the partial fraction coefficients of the last row of the tableau must be divided by 2 . The resultant partial fraction expansion becomes

$$
F(s)=\frac{-\frac{1477}{16}}{(s-1)}+\frac{-\frac{245}{8}}{(s-1)^{2}}+\frac{-\frac{21}{4}}{(s-1)^{3}}+\frac{-30}{(s-2)}+\frac{-\frac{321}{2}}{(s-2)^{2}}+\frac{\frac{2005}{16}}{(s-3)}
$$

Operation Count. In order to compare the efficiency of a method it is useful to have an expression for the number of multiplications and divisions required, the assumption being made that additions and subtractions are less troublesome. Let $\boldsymbol{m}$ $(<n)$ be the degree of the numerator. In order to find the $\beta$ coefficients, $(m)(m+1) / 2$ multiplications are required when Horner's scheme is used. From (21) and (22) it is seen that the native coefficients require no multiplications or divisions. According to (19) and (20), there is one division for each of the $n^{2} / 2-S / 2$ alien coefficients, where

$$
S=M_{1}^{2}+M_{2}^{2}+\cdots+M_{q}^{2} .
$$

In the nonmonic case $n$ additional division are required. The total operations count becomes

$$
\frac{n^{2}}{2}+n-\frac{S}{2}+\frac{(m)(m+1)}{2} .
$$

The last term may range from zero when $m=0$, up to $(n)(n-1) / 2$ when $m=n-1$. Hence, the operations count varies from a low value of $n^{2} / 2+n-$ $S / 2$ to a high value of $n^{2}+n / 2-S / 2$.

Henrici [2] gives the operations count for his method B as being less than $2 n^{2}+S$. The present author reanalyzed the method and, under the assumption that $(n+1) \geqslant 2 M_{i}, i=1,2, \ldots, q$, found that the low value for the operations count (for $m=0$ ) is $2 n^{2}+n-2 S$ and the high value (for $m=n-1$ ) is $3 n^{2}+\frac{1}{2} n$ $-\frac{5}{2} S$. Although a definitive comparison of the operations counts given above is difficult, it appears that the escalation method requires fewer than half as many multiplications and divisions as does method B of Henrici.

\footnotetext{
Department of Industrial and Systems Engineering

The University of Florida

Gainesville, Florida 32611
}

1. D. Hazony \& J. Riley, "Evaluating residues and coefficients of high order poles," IRE Trans. Auto. Cont., AC-4, 1959.

2. P. Henrici, Applied and Computational Complex Analysis, Vol. 1, Wiley, New York, 1974. 